\title{
Alterstice
}

Revue internationale de la recherche interculturelle

International Journal of Intercultural Research

Revista International de la Investigacion Intercultural

\section{Rapports entre " petites " et " grandes " langues : tensions et négociations identitaires - exemples dans divers contextes}

\section{Claudine Brohy}

Volume 2, numéro 1, 2012

Stratégies de (re)médiation en situation plurilingue. Études de cas en contextes de recherche et d'éducation

URI : https://id.erudit.org/iderudit/1077553ar

DOI : https://doi.org/10.7202/1077553ar

Aller au sommaire du numéro

Éditeur(s)

Alterstice

ISSN

1923-919X (numérique)

Découvrir la revue

Citer cet article

Brohy, C. (2012). Rapports entre " petites » et " grandes » langues : tensions et négociations identitaires - exemples dans divers contextes. Alterstice, 2(1),

55-66. https://doi.org/10.7202/1077553ar
Résumé de l'article

Dans cet article nous explorons des grandes et petites langues en contact, avec leurs enjeux identitaires et linguistiques. Les exemples sont essentiellement tirés du paysage linguistique suisse, où quatre langues nationales côtoient des dialectes, des langues de la migration et l'anglais. 


\section{구}

alterstice

Revue Internationale

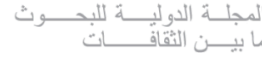

Revista International

de la Investigacion Intercultural

Revista Internacional

International Journal

de la Recherche Interculturelle

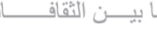

ARTICLE THÉMATIQUE

\section{Rapports entre " petites " et " grandes " langues : tensions et négociations identitaires - exemples dans divers contextes}

Claudine Brohy ${ }^{1}$

\section{Résumé}

Dans cet article nous explorons des grandes et petites langues en contact, avec leurs enjeux identitaires et linguistiques. Les exemples sont essentiellement tirés du paysage linguistique suisse, où quatre langues nationales côtoient des dialectes, des langues de la migration et l'anglais.

\section{Rattachement de l'auteure \\ ${ }^{1}$ Université de Fribourg, Fribourg, Suisse \\ Correspondance \\ claudine.brohy@unifr.ch}

\section{Mots clés}

langues en contact; politique des langues; identité

\section{Pour citer cet article :}

Brohy, C. (2012). Rapports entre "petites » et " grandes " langues : tensions et négociations identitaires exemples dans divers contextes. Alterstice, 2(1), 55-66. 


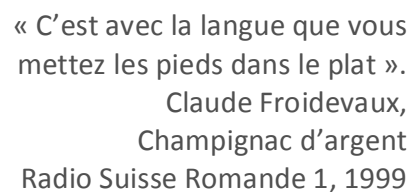

\section{Introduction}

Cette contribution tente de présenter des situations complexes, des lieux de rencontre ou d'exclusion entre des langues considérées comme, d'une part, importantes, légitimes, reconnues, et, d'autre part, des langues minorées, ou exclues, ou de portée restreinte. Toutefois, si l'on parle communément de rapports et de contacts entre langues, il s'agit en fait de locuteurs - ou plutôt d'utilisatrices et d'utilisateurs des langues en question, car I'utilisation des langues ne se limite pas à l'habileté à les parler - qui sont en contact, comme le soulignait déjà Weinreich dans les années 1950 : " [...] two or more languages will said to be in CONTACT if they are used alternatively by the same persons. The language using individuals are thus the locus of the contact " (Weinreich, 1970, p. 1).

Je vais présenter quelques exemples de rencontres entre utilisatrices et utilisateurs de différentes langues, dans des contextes multilingues divers, avec des langues qui véhiculent des valeurs et des cultures variées, dans lesquelles non seulement les identités très souvent composites sont négociées (Pavlenko et Blackledge, 2004), mais aussi le statut des langues, et donc aussi leur image en tant que petites ou grandes langues, avec toutes les connotations afférentes, ainsi que la subjectivité et relativité des concepts. En effet, une petite langue peut devenir ou être perçue en tant que grande langue et inversement. Des facteurs individuels, sociétaux et institutionnels sont en constante interrelation, et nous allons voir comment les individus et les institutions réagissent à la présence de plusieurs langues et quelles sont leurs stratégies pour limiter leur nombre et leurs champs d'action, ou au contraire, l'étendre et le diversifier (voir Alao, 2008). Les exemples sont essentiellement tirés du contexte suisse, avec quelques regards vers d'autres cas de figure.

\section{Langue, identité et individu}

Attardons-nous un moment à la notion d'identité qui va être essentielle pour la description des diverses situations de contact. Le terme d'identité est une notion transverse des sciences sociales: I'histoire, la philosophie, la psychanalyse, la psychologie sociale, l'anthropologie, la sociologie, l'ethnologie, la sociolinguistique et bien d'autres disciplines encore l'intègrent dans leurs méthodes et démarches scientifiques (voir Widmer, 2004).

Le terme identité traduit bien le fait de rester "idem " même dans des conditions - y compris les conditions linguistiques et culturelles - changeantes, mouvantes, dynamiques; celui d'individu véhicule la notion d'être indivisible. Malgré cela, l'identité est de plus en plus perçue comme étant une caractéristique hybride et bricolée (voir Moore et Brohy, sous presse) qu'il s'agit de faire accepter, qu'il faut adapter, négocier, construire en interaction avec son entourage proche ou lointain. Le langage, la langue et la parole (pour reprendre la triade saussurienne) sont largement associés à cette élaboration identitaire, et le choix d'une langue, d'une variété ou d'un registre est un acte identitaire (LePage et Tabouret-Keller, 1985) - souvent inconscient - qui inclut autant qu'il peut exclure :

Les langues (les dialectes, les accents, les variétés, les parlers bilingues, etc.) servent ainsi de marqueurs emblématiques des identités, une notion plurielle qui condense une série de significations et combine et imbrique construction de soi, sentiments d'appartenance et reconnaissance, impliquant donc des facteurs tant individuels que sociétaux. (Moore et collab., à paraître)

Toutefois, le rôle de la langue comme habit identitaire n'est pas forcément accepté par tout le monde. Une traductrice d'origine hollandaise, vivant en Suisse depuis son enfance, décrit de manière spontanée sa perception du binôme langue-identité :

Pour moi, une langue est uniquement un moyen d'expression et pas du tout un facteur d'identification (ce qui est sûrement compréhensible dans mon cas), je ne pourrais ainsi jamais m'engager dans un conflit linguistique comme celui qui couve 
actuellement à Fribourg. J'adapte les langues selon les circonstances; je passe du français à l'allemand, de l'anglais au hollandais tout en me sentant parfaitement moi. (Brohy, 1992, p. 319)

Une façon tout autre d'exprimer son identité plurilingue (ou plurielle) se rencontre dans le poème "Bilingual blues " :

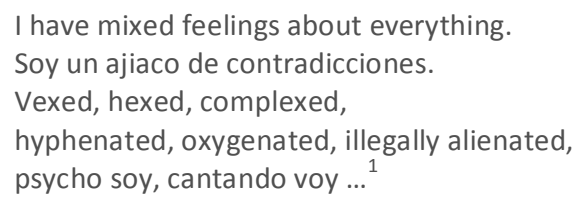

Dans un pays plurilingue comme la Suisse, l'identité n'est pas seulement discutée à l'intérieur du pays, par l'intermédiaire des communautés linguistiques, mais aussi par rapport aux hinterlands linguistiques respectifs que sont la France, l'Allemagne et l'Italie. Dans ces cas, même s'il s'agit de variétés proches, les différences culturelles et politiques sont importantes. Une forte immigration des pays voisins vers la Suisse et des flux frontaliers quotidiens rendent la cohabitation parfois difficile. Ainsi, durant les championnats de foot, des t-shirts rouges avec le slogan, écrit en forme de croix blanche, "Ich unterstütze zwei Mannschaften, die Schweiz und jedes Team, das Deutschland schlägt » (« Je soutiens deux équipes: la Suisse et celle qui battra l'Allemagne ») font fureur; on en trouve également en français par rapport à la France.

Nous allons mettre maintenant en relation les langues avec d'autres paramètres sociétaux et individuels, tels que le nombre de locuteurs, le degré de codification, les usages et les représentations.

\section{Langues, plurilinguisme et statuts des langues}

\section{Aspects quantitatifs}

Un moyen d'évaluer l'importance d'une langue est sans doute de jauger sa valeur numérique de par le monde. Si l'on se réfère aux statistiques linguistiques par utilisateur pour un choix de langues, on obtient le tableau suivant:

Tableau 1 : Importance numérique des langues

\begin{tabular}{clr} 
Rang & \multicolumn{1}{c}{ Langue } & Locuteurs \\
\hline 1 & Mandarin & 845 millions \\
2 & Espagnol & 329 millions \\
4 & Anglais & 328 millions \\
5 & Hindi & 242 millions \\
6 & Arabe & 182 millions \\
7 & Bengali & 181 millions \\
8 & Portugais & 178 millions \\
9 & Russe & 144 millions \\
10 & Japonais & 122 millions \\
16 & Allemand & 90 millions \\
$\ldots$ & Français & 68 millions \\
$\ldots$ & Romanche & 35 000 \\
Sources : Lewis (2009) pour la hiérarchie 1 à 16; Office fédéral de la statistique (Suisse) pour le \\
romanche; Filipi (s. d.) pour l'istro-roumain
\end{tabular}

Nous le voyons, le statut numérique des langues varie considérablement, ici entre presque un milliard d'utilisateurs pour le mandarin et seulement 250 pour l'istro-roumain, la langue des descendants des Valaques établis en Istrie (Croatie) dans quelque dix villages et dont le nombre de locuteurs diminue inexorablement, en passant par les 35'000 locuteurs pour le romanche, l'une des quatre langues nationales helvétiques, qui a aussi le statut d'une langue officielle partielle et dont il sera encore question plus tard. Toutefois, lors du comptage des locuteurs il faut

\footnotetext{
${ }^{1}$ Pérez Firmat, G., Bilingual Blues, 1995. www.gustavoperezfirmat.com/bilingual_blues.html [consult. le 14 août 2010]
} 
préciser s'il s'agit uniquement de locuteurs natifs (L1), ou si les bilingues ou plurilingues sont également répertoriés, ce qui est loin d'être toujours le cas dans les statistiques des langues. Pour calculer la force d'une langue, il vaudrait ainsi mieux compter le nombre de personnes qui l'apprennent en tant que langue seconde ou étrangère, ce qui traduit l'attractivité culturelle et économique d'une langue et peut-être son importance future. Ainsi, l'anglais n'est qu'en troisième position en tant que langue première, mais en première position en tant que langue seconde (L2), étant donné qu'il y a certainement plus d'un milliard d'individus qui l'apprennent en tant que L2. L'espagnol est une L1 importante, qui est également souvent apprise en tant que L2, L3 ou L4. En Suisse, par exemple, grâce à son prestige en tant que langue internationale, l'espagnol supplante l'italien en tant que L4, alors que l'italien est une langue nationale et la langue d'une communauté immigrée importante, même si elle va en diminuant.

\section{Aspects politiques}

Dans certains cas, la délimitation entre les langues peut poser problème pour calculer le nombre d'utilisateurs. Ainsi, les glossonymes utilisés dans le cadre du bosnien, du croate, du monténégrin et du serbe (BCMS) a été et est toujours un enjeu politique important. Même si la distance entre les langues (dans le sens de abstand selon Kloss, 1976) paraît minime, les langues respectives jouissent d'un ausbau (Kloss, 1976) confortable vu qu'elles sont les langues officielles des États-nations correspondants. Le fait que les différents États protègent les autres variétés linguistiques respectives dans le cadre de la Charte européennes des langues régionales ou minoritaires (Conseil de l'Europe, 1992) renforce encore cette distance symbolique.

\section{Aspects qualitatifs}

La charge émotive suscitée par les langues est également un facteur important. En plus de qualificatifs tels que belle, utile, importante, certaines langues (secondes) doivent remplir des fonctions de proximité sociale, voire d'amitié, on parle dès lors de langues partenaires et de langues du voisin (sans que cela implique des langues typologiquement proches). II faut donc apprendre les langues des amis, tout comme il faut s'approprier celles des ennemis, les anciens films d'espionnage nous montrant l'exemple d'agents ou de doubles agents qui trompent leur entourage par une compétence native de langues étrangères. Sans que nos concurrents soient nécessairement nos ennemis ou même nos adversaires, le discours ambiant nous dit qu'il faut apprendre les langues étrangères pour des raisons économiques, des langues qui présentent une plus-value. Il faut donc se démarquer par des compétences peu communes qui nous apportent face à nos concurrents un avantage sur le marché du travail, sans pour autant négliger des compétences en anglais. Néanmoins, il existe aussi une catégorie de langues critiques. Après les attentats du 11 septembre 2001, l'administration Bush a débloqué un budget de 112 millions de dollars pour le programme Critical languages et l'enseignement de langues telles que le coréen, l'ourdou, le pachtou et l'arabe, ceci afin de lutter contre le terrorisme international et pour redorer l'image des Etats-Unis à l'extérieur du pays (voir Brohy, 2007). Nous savons donc que les langues sont apprises pour des raisons variées, les plus fréquentes indiquées étant la culture, les vacances, l'amitié, la famille et le travail. II est discutable de penser que la lutte contre le terrorisme et l'amélioration de l'image soit une impulsion suffisante à l'apprentissage efficace des langues étrangères.

\section{L'anglais, une langue invasive?}

Parmi les grandes langues, on cite bien sûr la langue internationale par excellence, l'anglais, même si on veut souvent relativiser sa prépondérance en la noyant dans un groupe indéfini de langues dites « internationales ». On l'appelle également globale, of the new economy, lingua franca, le nouveau latin, langue de la science, ou, lorsqu'il s'agit d'une variété (hypothétiquement) simplifiée ou dénuée de culture, globish. L'appétence des grandes langues à absorber les petites est qualifiée de glottophagie (Calvet, 1974), de linguicism, de génocide linguistique (Skutnabb-Kangas, 2000) et même de linguicide (Skutnabb-Kangas et Phillipson, 1995), et l'anglais est donc logiquement qualifié de killer language, parce qu'il menacerait les autres variétés dans un environnement donné et qu'il représente une espèce invasive pour des langues indigènes, menant parfois à leur éradication complète (Dorian, 1981). Le poids de l'anglais face aux autres langues (voir Gasquet-Cyrus et Petitjean, 2009), ainsi que les raisons de son statut prépondérant, sont souvent évoqués, et cela depuis de nombreuses années :

Alterstice - Revue Internationale de la Recherche Interculturelle, vol. 2, $n^{\circ} 1$ 
Facteur [...] qui a fortement agi en faveur de l'anglais, aux dépens du français, la simplicité. L'anglais moderne est, en effet, facilement accessible, non seulement aux peuples européens, mais aux orientaux et extrême-orientaux. La grammaire est sommaire. Le vocabulaire, pratique et explicite. II a, sur le français, l'avantage de posséder une syntaxe facile; sur l'allemand et le russe, de ne connaître ni déclinaisons ni conjugaisons complexes. (Pigot, 1970, p. 19)

Dans un contexte ex-colonial, par exemple en Algérie, l'anglais est souvent considéré comme une langue neutre, par rapport au français qui véhicule des connotations de politique coloniale. En Suisse, où la discussion autour du choix d'une langue nationale ou de l'anglais comme L2 ou L3 à l'école a fait rage, les articles dans la presse ont révélé des associations telles que rural, analogique, vieux jeu pour le français, tandis que l'anglais est qualifié d'urbain, de digital et de moderne.

\section{Plurilinguisme et langues minoritaires}

À l'opposé, les très petites langues sont appelées Kleinsprachen (petites langues) ou même Kleinstsprachen (langues minuscules) et deviennent par la suite des langues menacées, puis moribondes, même après avoir été mises sous perfusion étatique grâce à des subventions. Cette personnalisation de l'état des langues se retrouve dans des termes associés tels que vitalité, longévité, mortalité, et aussi revitalisation, qui est parfois pratiquée avec succès dans le cadre de langues minoritaires, comme par exemple le gallois qui a connu une renaissance grâce à l'enseignement bilingue. Généralement, ces petites langues ne sont non seulement utilisées que par un petit nombre de personnes, mais elles sont aussi apprises par un nombre infime de personnes en tant que L2 ou L3 parce qu'elles n'ont que peu de valeur sur le marché du travail. Ainsi, les personnes parlant le finnois, le hollandais, le romanche ou le sorabe connaissent généralement plusieurs langues, étant donné que peu de monde apprend les leurs.

\section{Le dialecte alémanique : une langue qui a réussi}

Une langue ou une variété de langue, considérée pendant un certain temps dans un contexte social donné comme langue en danger, peut devenir une langue forte. C'est le cas du catalan, par exemple, réduit à une langue cachée et interdite sous l'ère de Franco, devenue la langue de la Catalogne (on utilise aussi le catalan dans la communauté valencienne, dans les Baléares, à Andorre et en Sardaigne) par une politique volontariste de normalisation, c'est-àdire de rendre son utilisation normale dans toutes les sphères de la vie. Dans le cas du dialecte alémanique (appelé aussi suisse-allemand, ou simplement dialecte, en réalité un ensemble de dialectes), variété utilisée dans la partie germanophone de la Suisse par toutes les couches de la population pour la plupart des échanges oraux et même pour certains textes informels et privés écrits (courriels, textos, cartes postales, journaux intimes, notes personnelles) ${ }^{2}$, on craignait sa disparition sous la pression de l'allemand écrit standardisé qui influença de plus en plus la langue orale en Allemagne. Le projet de conserver des traces de cet idiome et de ses nombreuses variétés pour les générations futures a mené à la publication du Schweizerisches Idiotikon, glossaire dont la conception remonte au début du $19^{\mathrm{e}}$ siècle, ouvrage qui n'est pas encore terminé à ce jour. En même temps, les dialectes et patois ont subi des interdictions à l'école. Si celles-ci ont été fatales aux patois romands, elles n'ont cependant eu aucune incidence sur l'utilisation des dialectes alémaniques ${ }^{3}$ :

L'emploi du patois est sévèrement interdit dans les écoles, la langue française et l'allemand grammatical (Schriftdeutsch) [= allemand écrit, représenté en lettres gothiques, CB] sont seuls admis dans l'enseignement. Les instituteurs veillent à ce que, en dehors de l'école et dans les conversations entre enfants, il en soit de même. (Canton de Fribourg, 1886, art. 171 du Règlement Général)

La relation entre dialecte alémanique et allemand standard est une des quatre situations diglossiques prototypiques (les autres sont : dialectes arabes/arabe classique, dhimotiki/katharevusa en Grèce, créole/Ifrançais) décrites par Ferguson (1959), selon lequel une variante dite haute (" high ») côtoierait une variante basse (« low »)

\footnotetext{
2 II existe aussi une vaste production littéraire et culturelle en dialecte (prose, poésie, pièces de théâtre, opéras, films, chants, etc.). Le dialecte alémanique est largement utilisé dans les médias électroniques, même pour les informations.

${ }^{3}$ Quelques cantons alémaniques ont voulu limiter l'usage du dialecte et renforcer celui de l'allemand standard, ce qui a mené à des votations populaires à Bâle et à Zurich. Dans les deux cas, les initiatives (référendums) pour l'usage exclusif du dialecte à l'école maternelle ont été acceptées en 2011.
} 
dans une complémentarité fonctionnelle relativement stable. Vu le prestige social dont jouit le dialecte en Suisse alémanique, cette distinction n'est toutefois pas applicable. Si l'on dit communément qu'un dialecte est une langue sans armée ni flotte, et qu'une langue est un dialecte qui a réussi, on peut dire que le dialecte est ici une langue qui a fort bien réussi! Ceci ne va pas sans causer des problèmes à la communauté francophone de Suisse qui est habituée au statut mineur, voire à la disparition, des patois et qui n'accepte pas facilement l'omniprésence des dialectes, alors que la langue enseignée dans les écoles romandes est l'allemand standard ${ }^{4}$, et que beaucoup de personnes nourrissent des sentiments négatifs envers les patois et les dialectes, sentiments hérités de longue date et partagés avec le voisin français :

Ces dialectes, s'ils possèdent une littérature qui n'est pas sans saveur, n'ont point d'orthographe, point de syntaxe commune, donc point de grammaire. Ils sont impossibles à codifier, par leur diversité même. Leur unité n'est point à faire. Elle serait du reste contraire à leur génie. (Diesbach, 1943, p. 27)

On ne s'imagine pas une langue, utilisée en Suisse alémanique par cinq millions de locuteurs qui se débrouillent très bien au niveau économique et culturel, sans grammaire ni syntaxe... Et plus récemment, de la part d'un journaliste romand renommé : «[...] La prise de conscience d’un véritable handicap linguistique. Et peut-être intellectuel, car ces sympathiques parlers locaux, si riches dans le vocabulaire campagnard, ne sont pas des outils raffinés de la pensée » (Pilet, 2006). L'expression vocabulaire campagnard est mal adaptée au paysage linguistique alémanique qui est caractérisé par l'urbanité et des variétés mixtes propres aux parlers des villes (fortes influences françaises, anglaises et balkaniques, par exemple). La relation entre l'allemand standard et le dialecte, l'apprentissage de l'allemand en Suisse romande et la prétendue non compétence des Alémaniques en allemand standard sont souvent thématisés dans des lettres de lecteurs et d'innombrables blogues:

Allors ça c'est la meilleure! Depuis quand les suisses-allemands parlent-ils allemand ? Les romands se mettront à apprendre l'allemand quand les suisses-allemand le parleront!

Quand un Suisse-allemand apprend le français et qu'il vient à Genève il a l'immense chance d'écouter du français, ce qui est loin d'être le cas pour un francophone qui se rend en Suisse-alémanique. Il y a même une journaliste qui s'est faite virer comme une malpropre parcequ'elle a osé parler le bon allemand !!!!! Honte à eux !!! ${ }^{5}$

Nous voyons donc que le dialecte alémanique jouit d'un statut particulier entre langue forte informelle au niveau national et langue faible au niveau international, ce qui n'est pas sans provoquer des frictions avec les francophones qui ont (encore) l'impression d'utiliser une langue à vocation internationale.

Notons que la Charte européenne des langues régionales ou minoritaires du Conseil de l'Europe (1992), qui a pour tâche de préserver les langues régionales ou minoritaires en tant que patrimoine culturel de l'Europe et d'étendre leur usage dans tous les domaines de la vie publique, sociale et économique, n'a pas pour mission de protéger des dialectes (ni les langues de la migration d'ailleurs). Toutefois, il est laissé aux pays qui ont ratifié la Charte de décider s'ils considèrent les variétés à protéger comme langue ou comme dialecte. La Charte a été ratifiée par 25 des 47 États membres du Conseil de l'Europe qui choisissent dans un menu de 98 mesures les 35 qu'ils veulent mettre en œuvre. Le texte présente un discours dépolitisé, on parle de langues et non de locuteurs, et malgré le thème évidemment plurilingue, toutes les discussions et séances du Comité d'experts de la Charte se déroulent en anglais.

\section{Le romanche, une langue sous perfusion?}

Le romanche, parlé uniquement dans le canton des Grisons, seul canton suisse officiellement trilingue, est devenu la quatrième langue nationale suisse par votation populaire en 1938 en réponse à la politique d'irrédentisme du fascisme italien qui avait la fâcheuse tendance à le considérer comme un dialecte de l'italien. C'est une langue aujourd'hui menacée, parlée par $0,5 \%$ de la population suisse et par $14,5 \%$ de la population du canton des

\footnotetext{
${ }^{4}$ Certaines écoles romandes proposent des cours facultatifs de dialecte, et des modules de dialecte sont parfois intégrés dans les cours d'allemand ou des activités d'éveil aux langues.

${ }^{5}$ Commentaire de « Genevois » sur le blogue de P. Weiss, 2007. http://pierreweiss.blog.tdg.ch/archive/2007/08/21/achtung-agen\%C3\%A8ve-l-allemand-prend-tout-son-temps.html [publié le 20 septembre 2007, consulté sur le blogue le 21 août 2008 ].
} 
Grisons ${ }^{6}$. Il s'agit d'une langue sans véritable centre, qui est en fait composée de cinq variétés écrites, appelées idiomes, plus des variétés intermédiaires orales. Dans le contexte suisse, cette langue est souvent oubliée, on parle de trois langues nationales au lieu de quatre, on la qualifie parfois de mélange entre l'italien et l'allemand ou même de la sorte: "Le romanche, qui serait un mélange de latin et de roumain, est parlé dans quelques vallées (les Grisons) »".

Depuis 1982, il existe aussi une variété planifiée, le rumantsch grischun $(R G)^{8}$, développée par le romaniste zurichois Heinrich Schmid (Gross, 1999; Schmid, 1982), intégration des trois idiomes écrits principaux, avec des apports des autres variétés. II s'agit donc d'une planification de corpus qui s'est accompagnée d'une planification de statut visant à pallier la faiblesse de son éclatement. Le RG est devenu en 2001 la langue politique et administrative du canton des Grisons, elle est aussi la langue utilisée par la Confédération pour les documents qui concernent les locuteurs de toutes les variétés du romanche. On a commencé à introduire le RG dans les écoles dès 2003 avec des supports pédagogiques écrits en RG. Cette mesure ne fait de loin pas l'unanimité. Si certaines associations sont convaincues que la démarche est susceptible de sauver cette langue fortement minorée ${ }^{9}$, d'autres sont d'avis, au contraire, que l'utilisation d'une langue planifiée et artificielle, qui n'est la langue première de personne et qui a été imposée, mène directement à la perte du romanche, que le médicament censé sauver le malade ne l'achève en quelque sorte. Le canton des Grisons règle l'utilisation officielle des langues dans une Loi sur les langues (Sprachengesetz, 2006), qui stipule dans son article 3 que les autorités cantonales et les tribunaux cantonaux doivent utiliser le RG. Toutefois, les personnes de langue romanche sont libres d'utiliser soit les idiomes soit le RG pour les contacts avec les institutions cantonales.

Dans les faits, toutes les personnes de langue romanche sont au minimum bilingues, le contexte scolaire, économique et culturel ne permettant pas l'utilisation exclusive du romanche. En ce moment, des discussions virulentes au sujet non seulement du RG mais aussi des langues à apprendre à l'école sont en cours dans les Grisons, certaines personnes issues des milieux économiques voulant remplacer l'apprentissage du romanche et de I'italien en tant que L2 par l'anglais, qui est actuellement enseigné comme L3 à l'école, en évoquant le peu d'utilité économique de ces langues en dehors d'une valeur folklorique. Les propos du directeur d'une multinationale établie dans le canton des Grisons et président d'une association de tourisme, envoyés par voie électronique à un nombre important de personnes, étaient sans équivoques. Les langues cantonales, à part l'allemand, ne serviraient à rien pour la communication internationale et la mobilité, il faut donc introduire l'anglais en tant que L2. En plus, la situation linguistique compliquée du canton empêcherait les chercheurs et les personnes bien formées de s'établir dans le canton. Il aurait en outre remarqué que les Romanches ne maîtrisaient pas bien l'allemand, fait qui est réfuté par nombre de chercheurs qui ont enquêté sur les compétences langagières des Romanches (voir par exemple Scoula cumünela bilingua da Samedan, 2010). Les discussions s'articulent autour des concepts de local et global, alors que dans les faits, les Romanches utilisent souvent plusieurs langues en parallèle pour leurs échanges, comme en témoigne le passage ci-après trouvé dans un blogue qui mélange allègrement le suisse-allemand, l'allemand, le romanche (variété orale du Val Müstair) et l'anglais (cool, easy), mais aussi des signes typographiques (émoticônes) et le cumul de voyelles pour exprimer une emphase (vil spaaaaaass, "profitez bien ", littéralement « beaucoup de plaisir »), style qui caractérise l'oralité écrite des technologies de la communication. La dernière phrase est caractérisée par un double changement de code (traduction: Merci beaucoup pour l'invitation, je viens très volontiers; grazia fitg per et jau vegn sont en romanche, d'lladig et sehr gärn en suisse-allemand).

The Schnacuzys

jenu! chunsch den es anders mal uf es bierli verbi ;)

25 February 2010 at 10:47

au mann, i cha nöd cho:-( vil spaaaaaass eu allnä!!! ;-)

25 February 2010 at 00:01

${ }^{6}$ Office fédéral de la statistique, Neuchâtel. www.statistique.admin.ch/ [consult. le 14 août 2010]

${ }^{7}$ Questions sur la francophonie européenne, 2002. http://ml.hss.cmu.edu/fol/fol4/modules/module4/F4M402/F4M40201.html [consult. le 14 août 2010]

${ }^{8}$ D'autres langues connaissent une planification de corpus, l'hébreu ou le bahasa indonesia, par exemple.

${ }^{9}$ Pour une vue d'ensemble sur les discours, mythes et idéologies entourant les idiomes et le RG, voir Coray (2008). Le titre de son ouvrage en dit long sur les connotations associées aux variétés du romanche: "De la Mumma Romontscha (= Maman romanche, métaphore utilisée pour le romanche en tant que langue maternelle) au bébé-éprouvette RG ». 


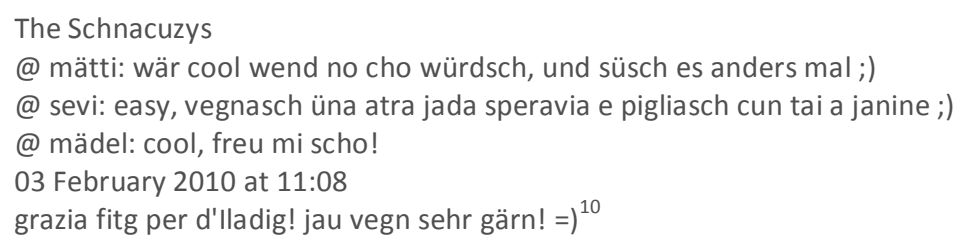

Les Romanches font donc preuve d'une utilisation des langues extrêmement polyglossique (diverses variétés de romanche, dialecte alémanique, allemand standard, souvent l'italien, le français et l'anglais, en plus des parlers plurilingues), le statut minoritaire du romanche les contraignant à s'adapter langagièrement. Malgré cela, une entreprise située à Coire, chef-lieu du canton, a envisagé d'interdire l'usage du romanche, étant donné que certains employés germanophones ne comprenaient pas leurs collègues...

\section{Le bilinguisme, une grande ou une petite langue?}

Le bilinguisme ou le plurilinguisme - définis ici comme une certaine aisance dans deux ou plusieurs langues, même si les compétences ne sont pas forcément natives ${ }^{11}$ et ne touchent pas tous les domaines de la même manière peut être perçu de manière positive ou négative, parfois dans le même contexte. En Suisse, une répartition subtile entre les locuteurs des régions linguistiques est de mise pour les contextes politiques, culturels et associatifs. II existe certes un droit linguistique codifié, il y a cependant aussi nombre de règles non écrites. Parfois, l'avantage linguistique d'être bilingue ou plurilingue est annulé par le handicap de ne pas pouvoir représenter légitimement une communauté linguistique de manière univoque ${ }^{12}$. Ceci a par exemple été le cas pour des élections au Tribunal fédéral ainsi qu'au Conseil fédéral (Gouvernement), l'art. 175 de la Constitution fédérale du 18 avril 1999 stipulant que « Les diverses régions et les communautés linguistiques doivent être équitablement représentées au Conseil fédéral ". Dans quelques cas, le bilinguisme des candidats a généré de nombreuses discussions et lettres de lecteurs au sujet de leur l'appartenance linguistique. II faut dire que les langues ne sont pas la chasse gardée des spécialistes en sciences du langage et des disciplines connexes. Cet état de fait n'est pas récent:

[...] le problème du bilinguisme n'est plus du ressort des seuls linguistes, psychologues ou pédagogues: le sociologue, le politicien, l'industriel, voire l'homme de la rue s'en sont saisi et prétendent pouvoir émettre des opinions à ce sujet, souvent, d'ailleurs, à bon droit et en connaissance de cause. Du coup, ce problème en a acquis une dimension nouvelle qui a permis des analyses originales et, partant, des réponses insoupçonnées et inattendues. Il arrive toutefois que ces avantages, procurés par l'éclatement du « domaine » du bilinguisme, soient annihilés par une méthode d'analyse impropre, c'est-à-dire non scientifique, ou par une information insuffisante au sujet des recherches et travaux qui ont déjà été consacrés à ce problème. (De Grève et Van Passel, 1970, p. 125)

Même si l'hypothèse dite pathologique associait autrefois systématiquement le bilinguisme avec toute une série de problèmes au niveau non seulement du langage, mais aussi de l'intelligence, des mœurs et du caractère - les bilingues seraient en fait des bilingoïdes aux contours identitaires et langagiers indéfinissables -, il arrive encore actuellement que le bilinguisme soit cité dans un contexte renvoyant à un problème de langage. Ainsi, en 2010, on fait mention à la radio romande d'un spectacle à Lyon monté avec des personnes qui ont des troubles du langage, c'est-à-dire des bègues, des aphasiques, des personnes souffrant d'Alzheimer et des ... bilingues. Aux Etats-Unis, durant les années 1990, lorsque les discussions autour de l'apprentissage des langues sous forme d'enseignement bilingue et d'immersion font rage, les termes de "bilingual students " et de "bilingual education " sont mis en relation avec des situations linguistiques problématiques et soustractives, alors que l'immersion jouit d'une aura positive.

\footnotetext{
${ }_{11}^{10}$ The Schnacuzys [blog]. http://de-de.facebook.com/pages/The-Schnacuzys/112408192133 [consult. le 14 août 2010]

11 Je suis bien consciente que cette définition n'est CECRiquement pas correcte, je reste toutefois dans cette approximation pour les besoins de ma contribution.

12 Officiellement, il n'y a pas en Suisse de bilinguisme individuel, le recensement fédéral ne permettant pas l'indication de plus d'une langue première (définie de la manière suivante: Quelle est la langue dans laquelle vous pensez et que vous savez le mieux?), par contre, I'utilisation de plusieurs langues dans certains domaines (famille, école, travail) peut être mentionnée.
} 
En situation multilingue, il y a également parfois déni de langue par rapport à des langues auxquelles on attribue moins de prestige. Ainsi, un étudiant africain (É) évoque de la sorte sa langue maternelle durant une entrevue faisant partie d'un test de classement au Centre de langues de l'Université de Fribourg:

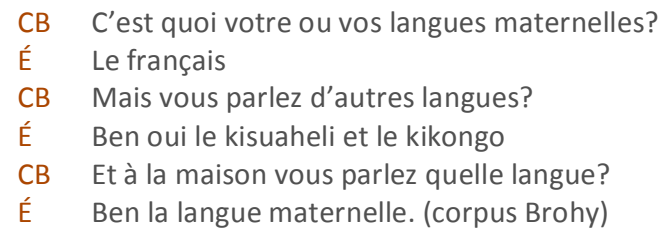

Cet étudiant n’a jamais mis un nom sur sa langue maternelle.

La cohabitation entre différentes langues dans une entité politique, comme cela est le cas dans des municipalités bilingues, génère des discussions par rapport aux normes et à la culture. Ainsi, un passant répond de la sorte durant un micro-trottoir effectué en 1972 à Bienne, ville officiellement bilingue, à la question: "Maintenant eeeh sur le plan culturel est-ce que vous pensez que Bienne ait disons une attitude particulière du fait de ce bilinguisme? »

Eeeh c'est peut-être un peu eeeh une ville qui n'a plus tout à fait le caractère ni romand ni suisse-allemand c'est une espèce de transition je vous dirais qu'en ce qui me concerne personnellement eeeh je suis un peu gêné de le dire mais Bienne me donne le sentiment eeeh de préfigurer dans le sens de l'avenir ces sociétés qui n'ont plus beaucoup de caractère propre mais qui vont dans un sens pratique vers une fusion de tout le monde n'est ce pas alors sous cet angle-là c'est un avantage mais évidemment pour les personnes qui sont rattachées à des valeurs culturelles eeeh celles-ci eu sont partiellement sacrifiées parce que je pense pas qu'on puisse superposer vraiment les cultures on ne peut pas se cultiver dans plusieurs langues. ${ }^{13}$

Nous voyons que la norme renvoie ici à une situation de monolinguisme, seule susceptible de générer des conditions culturelles favorables. 40 ans plus tard, les discours sont plus nuancés, il y a certes un fort ancrage idéologique en faveur de l'identité monolingue (mais pas en terme de compétences plurilingues), avec à l'autre bout du continuum des énoncés marqués pour le plurilinguisme, parfois tout aussi idéologiques.

\section{Bilinguisme et langues hybrides}

Si le bilinguisme en général crée des discussions intenses, une des situations observables les plus contestées des langues en contact est sans doute le mélange des langues. La ville bilingue de Fribourg connaît une langue hybride, autrefois largement pratiquée dans les quartiers défavorisés de la Basse-Ville, appelée bolze (voir Brohy, 1992, p. 81-83), un mélange de français, de dialecte alémanique et de patois. Ce parler (et ses locuteurs) ne rencontrait que du mépris de la part des habitants des hauts quartiers, il jouit aujourd'hui d'une sorte de renouveau et de contre-prestige, en lien avec les identités locales revisitées. Le bolze rappelle le chiac du Nouveau-Brunswick, sauf qu'il est une variété transmise dans le cadre familial et celui des amis, et pas dans celui de l'école, comme l'évoque une étudiante franco-canadienne dans sa biographie langagière :

À la maison, ils [mes parents] m'ont toujours strictement interdit de communiquer en utilisant les expressions 'chiac' qu'on apprenait à l'école. Le chiac est le langage populaire et familier chez presque tous les jeunes francophones de ma région. Celui-ci est composé d'un mélange de français et d'anglais. En voici un exemple typique: 'Va ouère chercher ton car, il fait pretty frette pis chu already late pour un meeting. Ça me drive nuts d'attendre dehors et de freezer. Veux-tu ouère hurrier up! (corpus Brohy)

Si le mélange des langues est toujours largement stigmatisé dans beaucoup de milieux, les spécialistes du langage y voient actuellement plutôt une expression de compétence langagière, ainsi qu'un signe tangible de cultures et d'identités hybrides.

\footnotetext{
${ }^{13}$ Bloesch, J.-D., Bienne Biel. Chronique d'une ville moyenne de Suisse / Chronik einer mittleren Stadt in der Schweiz, 1972. DVD, Bienne, W. Gassmann.
} 


\section{Conclusion}

Après l'évocation de quelques contextes linguistiques minoritaires et majoritaires en chassé-croisé, dans lesquels des dialectes auraient dû rester restreints, confidentiels ou disparaître, de petites langues font peur, des langues régionales, nationales et internationales se bousculent et le statut de personnes plurilingues est ambigu, il est temps de poser quelques questions par rapport à des sujets largement débattus, à savoir le lien entre écologie linguistique et écologie environnementale, entre langues et mobilité, entre langues en contact et en conflit, entre plurilinguisme individuel et institutionnel, entre les différents statuts des langues impliquées dans la construction du plurilinguisme.

En réponse à la première question, si les linguistes, didacticiens et autres personnes proches des sciences du langage s'engagent souvent pour des langues et cultures menacées et ont un discours appuyé en faveur de la diversité culturelle et linguistique, il n'en va pas forcément de même pour notre environnement naturel. Mais à quoi bon promouvoir des petites langues et des cultures menacées si leur environnement naturel ne permet pas ou plus leur survie dans des conditions décentes? La remarque suivante illustre ce propos:

ah bon t'es linguiste et tu t'occupes de langues ... t'es certainement pour le maintien des langues... mais bon... moi, je trouve que la disparition d'un minuscule petit insecte est à longue échéance certainement beaucoup plus tragique pour la diversité des espèces... et a beaucoup plus de conséquences que la disparition d'une langue. (corpus Brohy)

Une autre interrogation, partiellement en lien avec la première, concerne le concept de mobilité en lien avec le plurilinguisme et le discours généralement extrêmement positif qui l'entoure. Les textes fondateurs de la politique linguistique éducative mettent souvent en parallèle l'apprentissage des langues, la mobilité et l'employabilité. Toutefois, il ne faut pas perdre de vue qu'il existe plusieurs type de mobilités et que les connotations positives ne rendent pas compte des enjeux identitaires, familiaux, sociaux et culturels de trajectoires de mobilités forcées par un environnement économique et politique défavorable.

Le troisième questionnement concerne l'utilisation des termes "langues en contact » ou "langues en conflit », souvent évoqués comme une opposition. Nous avons vu que les langues ne sont pas en conflit, ce sont les personnes utilisant les langues qui peuvent l'être le cas échéant. Cependant, tout contact (et pas seulement le contact langagier) est susceptible de générer des conflits, ce qui compte au final est la façon dont les conflits sont traités et résolus, c'est-à-dire la culture de gestion des conflits, et non la présence ou l'absence de conflit en soi.

Un autre problème est en relation avec le terme polysémique de plurilinguisme. Les discours ne font souvent pas la différence entre le plurilinguisme individuel et institutionnel, même en Suisse où les deux phénomènes sont largement répandus, et même de la part de personnes pourtant impliquées dans la recherche sur le plurilinguisme. Il est évident que les notions d'identité et de culture, en lien avec des langues qualifiées de petites, ou d'importantes, ou de minoritaires, ou d'officielles, se trouvent configurées différemment selon si elles touchent des personnes ou des institutions. Le terme même est d'ailleurs ambigu, tant le plurilinguisme peut toucher des langues qui connaissent un aménagement curriculaire, donc des objectifs scolaires, ou alors des langues à l'extérieur de l'école et parfois vues comme une entrave au développement de la langue de scolarisation. Toutefois, même dans ce cas, certaines langues peuvent être valorisées dans un contexte social donné, grâce à la force d'un contre-prestige (covert prestige) social et économique par exemple. Les termes pourtant très souvent utilisés en lien avec le plurilinguisme dans le contexte du Cadre européen commun de référence pour les langues (Conseil de l'Europe, 2001), à savoir des compétences fonctionnelles et partielles, ne rencontrent souvent pas l'adhésion de personnes sur le terrain, pour elles les langues se trouvant dématérialisées d'aspects culturels et identitaires. C'est un point de vue à prendre en compte dans les recherches futures sur les identités multilingues.

\section{Références bibliographiques}

Alao, G. (dir.). (2008). « Grandes » et « petites » langues : pour une didactique du plurilinguisme et du pluriculturalisme. Berne : Lang (= Collection Transversales, 24).

Brohy, C. (1992). Das Sprachverhalten zweisprachiger Paare und Familien in Freiburg. Freiburg: Universitätsverlag. 
Brohy, C. (2007). Von kleinen, grossen und kritischen Sprachen. Freiburger Nachrichten, 3 mars.

Calvet, L.-J. (1974). Linguistique et colonialisme. Petit traité de glottophagie. Paris : Payot.

Canton de Fribourg (1886). Règlement Général des écoles primaires du canton de Fribourg. Bulle : Imprimerie Ackermann.

Conseil de l’Europe (1992). Charte européenne des langues régionales ou minoritaires. Strasbourg : Conseil de I'Europe.

Conseil de l'Europe (2001). Cadre européen commun de référence pour les langues : apprendre, enseigner, évaluer. Paris : Didier.

Coray, R. (2008). Von der Mumma Romontscha zum Retortenbaby Rumantsch Grischun. Rätoromanische Sprachmythen. Chur : Verlag Bündner Monatsblatt.

De Grève, M. et Van Passel, F. (1970). Quelques considérations sur le bilinguisme précoce. Revue de l'Institut de Sociologie, 1, 125-136.

Diesbach, F. de (1943). Vérité sur la Suisse. Genève : Le milieu du monde.

Dorian, N. (1981). Language death: The life cycle of a Scottish Gaelic dialect. Philadelphia : University of Pennsylvania Press.

Ferguson, C. A. (1959). Diglossia. Word, 15, 325-340.

Gasquet-Cyrus, M. et Petitjean, C. (dir.). (2009). Le poids des langues. Dynamiques, représentations, contacts, conflits. Paris : L'Harmattan.

Gross, M. (1999). Rumantsch Grischun : Planification de la normalisation. Bulletin suisse de linguistique appliquée, 69(1), 95-105.

Kloss, H. (1976). Abstandsprachen und Ausbausprachen. Dans J. Göschel, N. Nail et G. van der Elst (dir.), Zur Theorie des Dialekts (p. 301-322). Wiesbaden : Steiner Verlag.

LePage, R. et Tabouret-Keller, A. (1985). Acts of identity. Creole-based approaches to language and ethnicity. Cambridge : Cambridge University Press.

Moore, D. et Brohy, C. (sous presse). Identités plurilingues et pluriculturelles. Dans J. Simonin et S. Wharton (dir.), Sociolinguistique des langues en contact, modèles, théories. Dictionnaire encyclopédique des termes et concepts. Lyon : ENS Éditions.

Pavlenko, A. et Blackledge, A. (dir.) (2004). Negotiation of identities in multilingual contexts. Clevedon : Multilingual Matters.

Pigot, M. (dir.). (1970). Les langues étrangères. Leurs trois aspects : social, pratique, juridique. Paris : Denoël.

Pilet, J. (2006). Baragouins. L'Hebdo, 19 janvier.

Schmid, H. (1982). Richtlinien für die Gestaltung einer gesamtbündnerischen Schriftsprache : Rumantsch Grischun. Cuira : Lia Rumantscha.

Scoula cumünela bilingua da Samedan (2010). Konzept zur Förderung der romanisch-deutschen Zweisprachigkeit im Kindergarten und in der Volksschule von Samedan. Samedan : Miméo.

Skutnabb-Kangas, T. (2000). Linguistic genocide in education. Mahwah, NJ : Lawrence Erlbaum Associates.

Skutnabb-Kangas, T. et Phillipson, R. (1995). Linguicide and Linguicism. Dans R. Phillipson et T. Skutnabb-Kangas, Papers in European Language Policy (p. 83-91). Roskilde: Roskilde Universitetscenter, Lingvistgruppen.

Weinreich, U. (1970). Languages in contact. Findings and problems ( $7^{\mathrm{e}}$ éd.). The Hague : Mouton.

Widmer, J. (2004). Langues nationales et identités collectives. L'exemple de la Suisse. Paris : L'Harmattan. 


\section{Sources citées}

Bloesch, J.-D. (1972). Bienne Biel. Chronique d'une ville moyenne de Suisse. Chronik einer mittleren Stadt in der Schweiz. DVD. Bienne: W. Gassmann.

Brohy, C. Langues et plurilinguisme à Fribourg (corpus Brohy, publication en préparation).

Constitution fédérale de la Confédération suisse du 18 avril 1999. http://www.admin.ch/ch/f/rs/c101.html

Weiss, P. [blogue] (2007). http://pierreweiss.blog.tdg.ch/archive/2007/08/21/achtung-a-gen\%C3\%A8ve-Iallemand-prend-tout-son-temps.html [commentaire de « Genevois », publié le 20 septembre 2007, consult. le 21 août 2008]

Lewis, M. (dir.). (2009). Ethnologue: Languages of the world (16 éd.) [en ligne]. Dallas : SIL International. www.ethnologue.com [consult. le 14 août 2010]

Filipi, G. (s. d.). Istrorumänisch. www.uni-klu.ac.at/eeo/Istrorumaenisch.pdf/ [consult. le 14 août 2010]

Office fédéral de la statistique. Neuchâtel. www.statistique.admin.ch/ [consult. le 14 août 2010]

Pérez Firmat, G. (1995). Bilingual Blues. www.gustavoperezfirmat.com/bilingual_blues.html [consult. le 14 août 2010]

Questions sur la francophonie européenne (2002). http://ml.hss.cmu.edu/fol/fol4/modules/module4/F4M402/ F4M40201.html [consult. le 14 août 2010]

Schweizerisches Idiotikon. (s. d.). Wörterbuch der schweizerdeutschen Sprache [en ligne]. Frauenfeld : Huber. www.idiotikon.ch. [consult. le 14 août 2010]

Sprachengesetz des Kantons Graubünden (SpG). (2006). www.gr-lex.gr.ch/frontend/versions/238 (19 octobre 2006 , consult. le 14 août 2010).

The Schnacuzys [blog]. http://de-de.facebook.com/pages/The-Schnacuzys/112408192133 [consult. le 14 août 2010] 